

ARTIGO ORIGINAL

\title{
Casa de apoio: suporte às mulheres com câncer de mama
}

\author{
Support house: support for women with breast cancer
}

\section{Casa de apoyo: apoyo a las mujeres con cáncer de mama}

Kuhn, Carla Inês ${ }^{1}$; Simionato, Débora Góes da Silva²; Bortoli, Cleunir de Fátima Candido de ${ }^{3}$; Coelho, Joeci Baldin Amorim ${ }^{4}$

Como citar este artigo: Kuhn CI, Simionato DGS, Bortoli CFC, Coelho JBA. Casa de apoio: suporte às mulheres com câncer de mama. J. nurs. health. 2018;8(3):e188310

\section{RESUMO}

Objetivo: conhecer o suporte oferecido às mulheres com câncer de mama em uma casa de apoio. Métodos: estudo qualitativo, descritivo e exploratório, realizado em uma casa de apoio às pessoas com câncer. Participaram do estudo seis mulheres em tratamento para câncer de mama. Os dados foram coletados pelo grupo focal e analisados pela análise de conteúdo. Resultados: o enfrentamento do câncer de mama após o diagnóstico é marcado pelo medo e insegurança, sendo a casa de apoio como suporte social e psicológico, representando confiança e fortalecimento para o tratamento. Considerações finais: a casa e grupos de apoio, a equipe de enfermagem, amigos e familiares são importantes suportes para contribuir com um bom prognóstico da mulher que vive com câncer de mama.

Descritores: Neoplasias da mama; Enfermagem em saúde comunitária; Apoio social.

\begin{abstract}
Objective: to know the support offered to women with breast cancer in a support house. Methods: qualitative, descriptive and exploratory study, performed in a house support for people with cancer. Participated in the study six women undergoing treatment for breast cancer. The data were collected by focus group and analyzed by the content analysis. Results: the confrontation of breast cancer after diagnosis is marked by fear and insecurity, and the house support, as social and psychological support, representing trust and strengthening for the treatment. Finals considerations: the support house and support groups, nursing staff, friends and family are important supports to contribute with a good prognosis of women living with breast cancer.
\end{abstract}

Descriptors: Breast neoplasms; Community health nursing; Social support.

\section{Resumen}

Objetivo: conocer el soporte ofrecido a las mujeres con cáncer de mama en una casa de apoyo. Métodos: estudio cualitativo, descriptivo y exploratorio, realizado en una casa de apoyo para las personas con cáncer. Participó en el estudio de seis mujeres que reciben tratamiento para el cáncer

1 Enfermeira. Faculdade de Pato Branco (FADEP). E-mail: carlaik95@hotmail.com http://orcid.org/0000-00023333-1557

2 Enfermeira. Faculdade de Pato Branco (FADEP). E-mail: debigois@hotmail.com http://orcid.org/0000-00031045-7541

3 Enfermeira. Mestre em Enfermagem. Faculdade de Pato Branco (FADEP). E-mail: cleunir_candido@hotmail.com http: / /orcid.org/0000-0002-1266-5267

4 Enfermeira. Especialista em Educação Profissional; em Materno-Infantil e em Nefrologia. Faculdade de Pato Branco (FADEP). E-mail: joeci@fadep.br http://orcid.org/0000-0002-1260-4608 


\section{JOURNALOF \\ Iss $2236-1987$}

de mama. Los datos fueron recogidos por el grupo de enfoque y analizados por análisis de contenido. Resultados: el enfrentamiento del cáncer de mama después de diagnosis está marcada por el miedo y la inseguridad y la casa de apoyo como apoyo social y psicológico, que representa la confianza y el fortalecimiento para el tratamiento. Consideraciones finales: los casa y grupos de apoyo, personal de enfermería, amigos y familia son importantes ayudas a contribuir con un buen pronóstico de las mujeres que viven con cáncer de mama.

Descriptores: Neoplasias de la mama; Enfermería en salud comunitaria; Apoyo social.

\section{INTRODUÇÃO}

0 câncer de mama é considerado o tipo de câncer mais frequente entre as mulheres no Brasil, exceto o câncer de pele não melanoma. Acomete principalmente as mulheres com idade acima de 35 anos, sendo considerado um dos principais problemas de saúde. - Brasil é um país com grande incidência de câncer de mama, estimativas do apontam 59.700 casos novos da doença, a cada ano em 2018 e 2019, estimando um risco 56,33 casos a cada 100 mil mulheres. ${ }^{1-2}$

Para que a doença se desenvolva, ela depende de alguns fatores de risco que contribuem para seu aparecimento. Estes podem ser genéticos, ambientais, reprodutivos, nutricionais, físicos, históricos, bem como tem relação com a duração da amamentação, ter obesidade, obesidade após a menopausa, tabagismo, alcoolismo, exposição às radiações ionizantes, níveis socioeconômicos, menarca precoce, nuliparidade, idade maior de 30 anos na primeira gestação, uso de contraceptivos hormonais, menopausa tardia e terapia de reposição hormonal. ${ }^{3-4}$

Os principais sinais e sintomas deste câncer são o nódulo fixo, endurecido e indolor, pele da mama avermelhada retraída, alteração no mamilo, aparecimento de pequenos nódulos nos braços e axilas ou pescoço, e presença de secreção nos mamilos. ${ }^{5}$ Para o diagnóstico da doença, a mamografia é apontada como o exame de escolha, com especificidade em torno de $90 \% .{ }^{6}$ No Brasil, os programas de rastreamento da doença, foram introduzidos tardiamente e com coberturas heterogêneas entre as diferentes regiões do pais. De acordo com o Instituto Nacional de Câncer (INCA), entre o período de 1995 e 2002, o rastreamento mamográfico em 15 capitais e Distrito Federal oscilou entre $37 \%$ e $76 \%$. Neste mesmo período, o Sistema Único de Saúde (SUS), realizou entre $17 \%$ e $54 \%$ do total de exames esperados, o que justifica em parte, o diagnóstico tardio e as elevadas taxas de mortalidade. ${ }^{7}$

Ao vivenciar o diagnóstico de câncer de mama, a mulher enfrenta muitas adversidades, como o tratamento que resulta em inúmeras modificações, tanto fisiológicas, quanto sociais e psicológicas. Mesmo diante de grandes avanços ao que se refere ao tratamento quimioterápico, as queixas relacionadas aos efeitos colaterais, durante as sessões de quimioterapia, são as mais frequentemente levantadas pelas pacientes. $E$ nesta conjuntura, 0 enfermeiro é um dos principais profissionais envolvidos com o tratamento das mulheres portadoras do câncer de mama, atuando 


\section{JOURNALOF \\ NURSING \\ ${ }^{\text {AND HEALTH }}$ \\ ISSN 2236 - 1987}

diretamente nos cuidados prestados, auxiliando as pacientes no enfrentamento do tratamento. ${ }^{8}$

Frente ao diagnóstico do câncer de mama, a rede de apoio constituída por familiares, amigos, desconhecidos e profissionais, são elementos essenciais no enfrentamento da doença e reabilitação da mulher. Para isso, a equipe multidisciplinar ao assistir a mulher deve ampliar o campo de atuação, contemplando o contexto social em que a mesma está inserida, estimulando a participação da família e demais relações significantes, como forma de estímulo no enfrentamento da doença. ${ }^{9-10}$

Uma rede social funcional presente, reflete em melhores condições de vida às pessoas, especialmente em casos de indivíduos com câncer. Ao fornecer o apoio em momentos de crise, a rede contribui para uma melhor autoestima e também aumento na qualidade de vida dos mesmos. Ademais, a ausência de redes sociais atuantes, resultam em uma experiência difícil, durante todo 0 processo de tratamento. ${ }^{11}$

Diante do exposto, este estudo teve por objetivo conhecer o suporte oferecido às mulheres com câncer de mama em uma casa de apoio.

\section{MATERIAIS E MÉTODOS}

Trata-se de um estudo qualitativo, descritivo e exploratório realizado em uma casa de apoio, denominada Grupo de Apoio à Mama (GAMA), localizada no município de Pato Branco, Paraná. O GAMA acolhe pacientes e os familiares que necessitam de auxílio, estes podem ser da cidade ou da região, onde recebem acompanhamento, orientações, atenção, moradia e alimentação durante o tratamento.

O local foi fundado por um grupo mulheres que vivenciaram o câncer de mama, com objetivo de acolher e confortar pacientes portadores de câncer que necessitam de apoio durante o tratamento. 0 serviço ofertado é gratuito, e disponibiliza moradia, alimentação, transporte da casa ao Hospital do Câncer, além disto, oferece oficinas de confecção manual, artesanato, promove reuniões com pacientes que estão ou passaram pelo tratamento, como a "roda de superação", procurando sempre proporcionar bem-estar à paciente.

Este estabelecimento é mantido por doações de empresários, de particulares, da comunidade e por meio de eventos promovidos pelas entidades e pelo próprio GAMA. A casa tem capacidade para receber 44 pessoas e fornecer até 80 refeições, sendo que se necessário, acolhe juntamente ao paciente debilitado um acompanhante para auxiliá-lo.

As participantes do estudo foram seis mulheres acolhidas na casa de apoio GAMA, que foram convidadas. Os critérios de inclusão foram: ter o diagnóstico do câncer de mama e estar frequentando a casa de apoio. Mulheres sem condições físicas e/ou psicológicas foram excluídas do estudo.

Os dados foram coletados no mês de maio de 2017, utilizando-se da técnica de grupo focal. Foram realizados dois encontros, o primeiro com duração de uma hora e o segundo 


\section{IOURNALOF

com duração de uma hora e trinta minutos.

Para aplicar a técnica, foram preparados e organizados os encontros a partir de um roteiro, com algumas questões orientadoras acerca da temática, como: o que lhe impulsionou a procurar um profissional de saúde? Como foi receber o diagnóstico do câncer de mama e quais as decisões foram tomadas? Quais os sentimentos presentes no diagnóstico e tratamento da doença? Qual o significado do câncer de mama antes $e$ após o diagnóstico? Quais os fatores determinantes no enfrentamento da doença? Qual a importância da casa de apoio GAMA durante o período de tratamento? O roteiro foi utilizado com flexibilidade e ajustado ao longo dos encontros.

Os encontros foram organizados e operacionalizados por duas acadêmicas do nono período do curso de Enfermagem da Faculdade de Pato Branco (FADEP), uma desempenhando a função de moderadora e a outra de observadora. A moderadora introduziu a temática, apresentou as questões orientadoras e facilitou a discussão. Já a observadora, participou da condução dos encontros, controlando o tempo, monitorando os gravadores, observando e registrando o início das falas das participantes.

A análise de dados deu-se por meio da técnica de análise de conteúdo temática, pela proposta operativa, ${ }^{12}$ que está dividida em duas fases, a exploratória e a interpretativa. Na fase exploratória, foram tratados aspectos fundamentais dos dados construídos, buscando a compreensão do contexto do grupo investigado. A análise na fase interpretativa buscou-se nos relatos das participantes 0 sentido, as projeções e as interpretações. Nesta fase ocorreu a ordenação e a classificação dos dados coletados, de forma horizontal através de leituras flutuantes do material. $\mathrm{Na}$ forma transversal, onde os dados coletados foram separados por temas ou categorias, facilitando a compreensão e interpretação dos dados coletados. $\mathrm{Na}$ análise final, constituiu o aprofundamento do material e na redação do relatório final.

A pesquisa respeitou todos os princípios éticos, assegurados de acordo com a Resolução 466/12 do Conselho Nacional de Saúde. ${ }^{13}$ Foi entregue um Termo de Consentimento Livre e Esclarecido disposto em duas vias, após assinados, uma via ficou com a participante e a outra com as pesquisadoras, o mesmo foi feito com o Termo de Consentimento para uso da imagem e voz. O anonimato das participantes foi garantido por um sistema alfanumérico, representado pela letra $M$, seguido de um número arábico, de acordo com a ordem de manifestação durante 0 grupo. 0 estudo foi submetido e aprovado pelo Comitê de ética e Pesquisa, sob número de Certificado de Apresentação para Apreciação Ética (CAAE) 66876417.2.0000.0116.

\section{RESULTADOS E DISCUSSÃO}

Na análise de conteúdo temática, do grupo focal com mulheres, emergiram duas categorias: enfrentamento do câncer de mama após o diagnóstico; e a Casa de apoio como suporte social e psicológico. 
Enfrentamento do câncer de mama após o diagnóstico

Muitas vezes, a descoberta do câncer é considerada uma sentença de morte, sentimentos contraditórios desabrocham, como negação, raiva, aceitação, e a incerteza do que vai acontecer, desperta um emaranhado de emoções, que até mesmo as mulheres sentem dificuldade em relatar. Neste momento é necessário o apoio e influência familiar e social, levando em consideração o contexto sociocultural que a paciente está inserida, pois interferem diretamente no bem-estar físico, funcional e emocional. ${ }^{14}$

As participantes do estudo, relataram sentimentos de superação no enfrentamento da doença. Mesmo que, logo ao diagnóstico a incerteza foi imperante, motivações como os filhos e a família, estavam presentes:

Descobri o câncer em casa durante $o$ banho com $o$ autoexame, senti a pontinha de um carocinho como se fosse um grão de arroz. Quando foi confirmado o diagnóstico, pensei que fosse uma sentença de morte, mas quando você passa por esta situação, nota-se que é apenas uma questão de tempo, $o$ tempo que é descoberto, o tempo de tratamento e a força de vontade é o que faz a diferença, mas é muito relativo, cada caso é um caso. (M3)

Percebi que meu braço começou a inchar, e notei um nódulo na mama direita bem duro, fui no médico e com os exames foi confirmado o câncer. Eu tinha medo de morrer, mas coloquei em minha cabeça que não podia, pois tinha dois filhos pequenos para criar um com dez e outra com um ano e pouco, isso me fortaleceu muito. (M1)

Com a confirmação do diagnóstico de câncer de mama, algumas mulheres apresentam sinais de alterações psicológicas, como trauma momentâneo, perda da autoestima, angústia e incerteza, fazendo com que ela mude seu comportamento e atitudes, em relação a sua família, meio social, e até mesmo pessoal. ${ }^{15}$ Observando isto, pôde-se perceber a partir dos relatos, que a família é o maior incentivo para uma boa recuperação, onde os pais, filhos, esposo, irmãos, e outros, servem como apoio fundamental para melhora acentuada da paciente, auxiliando nas atividades diárias e incentivando a realização do tratamento.

O médico me falou: 'a senhora tem câncer', mas me disse assim na lata! Eu me agarrei num negócio que tinha no meu lado, aquilo pareceu que o chão afundou, eu pensei 'o que vai ser de mim agora?' fiquei muito abalada. Quando recebi o diagnóstico e fui informada que precisava fazer a cirurgia, fui para casa e nos reunimos, eu, minha filha e meu marido, e no mesmo dia voltamos no consultório para marcar a cirurgia. Minha família me apoiou! (M4)

Em primeiro lugar o fator que determinou minha recuperação 


\section{IOURNAIOF

foi a minha família, porque eles me dão apoio. (M6)

Aos relatos, apesar das manifestações negativas, foi possível identificar atitude positiva, na qual a fé e a força de vontade servem de incentivo para seguir com 0 tratamento, assim obtendo ânimo e coragem para enfrentar a doença. 0 contato com outras pessoas em situação semelhante ou pior que a sua, abalam psicologicamente essas mulheres, mas também servem de motivação para seguir com 0 tratamento.

Um fator determinante é a fé e a força de vontade, são as duas partes, a espiritual e psicológica, que fazem a diferença que te colocam para cima, para fazer as quimioterapias e radioterapia sem reclamar. Aí você vê que tem gente em situação bem pior que a sua, que teu problema perto dos outros é mínimo. Tem gente que está lá todo retalhado de cirurgia, tem uns que chegam de maca, que estão vegetando, para fazer uma quimioterapia ou radioterapia. (M3)

Após a descoberta e o tratamento do câncer, o abalo emocional é acentuado, percebe-se que 0 meio social e as amizades são essenciais para uma boa recuperação. 0 reforço dos laços familiares e de amizade, serve de amparo à pessoa, que se sente fragilizada e precisando de apoio.

Nessas horas a gente vê quem realmente é amigo de verdade, que te ajudaria da mesma forma que você ajudaria. Diferente de algumas pessoas que faziam anos que eu não via, que não tinham mais contato e apareceram na minha porta, assim do nada para me dar um abraço, para me ajudar. (M3)

No tema abordado, é perceptível que a assistência integral à mulher neste período ofertada pelos familiares, amigos, grupos sociais, equipe de saúde, permite que ela venha a ter melhor qualidade de vida, pois estes fatores influenciam diretamente no bem-estar físico e psicossocial. ${ }^{16}$ Diante de um momento de angústias e inseguranças frente ao tratamento, o apoio familiar torna-se fundamental para o enfrentamento deste momento. ${ }^{14}$

\section{Casa de apoio como suporte social e psicológico}

O impacto causado pelo câncer de mama é vivenciado de diferentes maneiras por cada mulher. Envolve a existência de sentimentos e conceitos contraditórios, o que eventualmente resultam em conflitos a serem enfrentados por elas. Entre os sentimentos negativos, podem surgir distorções de autoimagem, da sexualidade e as incertezas sobre $o$ futuro. Por outro lado, a ressignificação da vida e o apoio familiar, estão entre os aspectos positivos que afloram neste momento. ${ }^{17}$

Diante deste contexto peculiar vivenciado pela mulher, contar com o acolhimento de uma casa de apoio é de suma importância no pré, durante e após o tratamento. Nos resultados deste estudo, as participantes revelam - papel da casa de apoio no enfrentamento da doença, atuando 
como suporte social, oferecendo informações sobre o diagnóstico de câncer de mama, tratamento e a reabilitação que o mesmo exige, além de moradia, alimentação e locomoção, para alguns casos em específico.

Eu estou morando aqui [na casa de apoio], mais para frente quero arrumar um emprego. Aqui, estão me ajudando, agradeço por tudo. Durante o tratamento de quimioterapia e radioterapia passo mal, comer não consigo, mas elas me ajudam aqui no que eu preciso. (M5)

Diferentes realidades revelaram o papel das redes comunitárias de apoio social no enfrentamento do câncer de mama, como de fundamental importância, levando em consideração as suas repercussões biopsicossociais. ${ }^{17-18}$ De uma forma em especial, grupos de apoio constituídos e liderados pelas próprias mulheres que vivem ou viveram a experiência do câncer de mama possuem a finalidade de estimular o protagonismo e a autonomia da mulher, de forma a assumirem o controle sobre suas próprias vidas. ${ }^{19}$

Meu marido procurou a casa para ter mais informação, porque não tinha ninguém na família que tinha passado por isso. Não sabia a rotina, quais os cuidados, porque é muito informação ao mesmo tempo. E como a casa foi fundada por mulheres que tiveram câncer de mama $e$ passaram por isso, me ajudaram amenizar um pouco os sintomas da quimioterapia. (M3)
Meu Deus! É assim, vem aqui na casa, conversa com uma, conversa com outra, é uma coisa importante. A gente vê pacientes que já estão se tratando, que ficam aqui na casa e também para ouvir quem já passou pelo tratamento. A casa aqui, é um lugar muito bom. (M6)

Um grupo de apoio proporciona importante atuação na vida das pacientes com câncer de mama. Mesmo diante de uma percepção negativa frente ao diagnóstico, a rede de apoio social, como o grupo de apoio, auxilia no enfrentamento da doença de maneira a dar suporte e esperança durante 0 tratamento. 0 grupo possibilita um espaço para compartilhar informações, conhecimento, experiências, assim como a participação em projetos desenvolvidos, e em campanhas e eventos com o objetivo de divulgação de informações a respeito do câncer de mama. ${ }^{14}$

Além dos desafios advindos do diagnóstico e tratamento do câncer de mama, muitas mulheres vivenciam as modificações na imagem, oriundas principalmente em decorrência da mastectomia. Porém o empoderamento da mulher, promovido pelo suporte social, ameniza essas modificações físicas, que afetam a autoimagem da mulher.

Eu só me arrependo de não ter colocado a prótese. 0 médico perguntava se eu queria colocar, e eu dizia não estou preparada no momento, quem sabe amanhã eu venha aqui e diga para o senhor vamos colocar. E não coloquei, $e$ agora não tem mais como fazer. 


\section{JOURNALOF \\ NURSING \\ ANO HEALTH}

Mas o que importa é estar bem e estar viva. (M1)

Diante dos traumas que a quimioterapia e radioterapia deixam no corpo, a casa propõe atividades que ajudam diretamente as pacientes, promovendo reuniões mensais (roda de superação), atendimento individual com psicólogos, conta com voluntários que ofertam serviços de estética como manicure, pedicure, maquiagem, e também outras oficinas de lazer como artesanatos, canto, leitura, e outras atividades de distração.

As contribuições terapêuticas do grupo de apoio, são evidenciadas em estudo, que aponta o momento de expectativas de alívio frente as dificuldades vivenciadas, despertando o otimismo e aumentando a confiança em relação ao futuro. A esperança se faz presente, quando um identifica a melhora do outro, vislumbrando o progresso do seu próprio tratamento, como uma possibilidade concreta. ${ }^{20}$

Exposta a importância da casa em relação ao auxílio do tratamento como suporte social e psicológico, as mulheres citam a importância que a casa tem em suas vidas apoiando de forma afetiva e elevando a autoestima, e fornecendo acompanhamento com fisioterapia o que diminui as restrições que o câncer de mama e o tratamento causaram.

A casa é muito boa, porque comecei a fazer as fisioterapias, que ajudaram a melhorar meu braço que estava muito inchado e dolorido. Além de todo apoio e ajuda prestado pela equipe da casa, que com certeza me ajudaram neste momento difícil. (M1)

É muito bom para mim, é como minha casa. Eu gostei porque me atendem bem, faço fisioterapia e não tenho o que reclamar, nota dez para a casa. (M4)

Aos relatos das pacientes é notável que elas se sentem bem em frequentar a casa, pois é um local onde a troca de experiências e a convivência com pessoas com a realidade similar a sua, as tornam mais fortes, neste sentido algumas mulheres com desejo em auxiliar o próximo a passar por esta fase difícil que elas já passaram, se tornam voluntárias na casa promovendo o bem-estar aos demais pacientes atendidos.

A vivência das mulheres em um grupo, pautado no compartilhamento de informações, no acolhimento, na busca do alívio emocional, na solidariedade e em atividades ocupacionais e recreativas, possibilita um combate ativo da doença. A possibilidade de compartilhar suas experiências, construir novas amizades e ressocialização, resultam no fortalecimento das mulheres, na luta contra a doença e ressignificando suas concepções e atitudes frente ao câncer. ${ }^{19}$

A participação em grupos de apoio apresenta muitos benefícios a pessoa com câncer, entre eles a diminuição dos níveis de desesperança, da preocupação, da ansiedade e do estresse, além do fortalecimento do funcionamento social das participantes. ${ }^{21}$ Conviver nestes espaços, permite as mulheres compartilhar suas experiências, 


\section{ISSN 2236 - 1987}

receber e oferecer suporte, sair da exclusão social, além de acessar informações sobre a doença e de suas formas de tratamento. Com isto, possibilita um ajustamento psicossocial de suas integrantes frente a doença, redescobrindo a força para viver, possibilitando a mulher, ser um agente multiplicador de informações sobre a doença. ${ }^{22}$

\section{CONSIDERAÇÕES FINAIS}

0 desenvolvimento deste estudo possibilitou conhecer o suporte ofertado pela casa de apoio às mulheres com câncer de mama, tal como: moradia, alimentação e transporte, bem como atividades complementares, que influenciam diretamente no bem-estar e na autoestima, resultando num melhor prognóstico à paciente.

A participação social é fundamental na recuperação da mulher. No grupo de apoio presente na casa, as pacientes têm a oportunidade de conviver e compartilhar experiências com pessoas que tem ou tiveram problemas similares. Com isto, identificaram meios que as auxiliam numa boa recuperação, sentem-se mais seguras e determinadas para realizar o tratamento, demonstrando entusiasmo, dedicação e força.

A família e os amigos também são de extrema importância, desde a confirmação do diagnóstico, pois servem de alicerce à paciente, buscando informações, auxiliando nos afazeres e juntamente com grupo de apoio. São essas pessoas que irão estimular e fortalecer psicologicamente a mulher, fortalecendo o enfrentamento da doença.

Em relação às pacientes, pode-se perceber que estas sentem prazer em frequentar a casa, não como obrigação de participação a dar continuidade ao tratamento, mas como meio de convivência agradável, pois são criados vínculos de amizades, e como a casa de apoio é mantida com a ajuda de voluntários, algumas se tornam voluntárias, devido à vontade de ser solidário ao próximo e compartilhar sua experiência como forma motivacional. E nestes casos, elas não estão fortalecendo apenas os ouvintes, mas a elas mesmas.

Com este estudo é possível sugerir que a casa, proporciona um espaço de atuação para a enfermagem e também, por meio da instituição de ensino superior, com a presença dos acadêmicos para o desenvolvimento de atividades relacionadas à função, auxiliando, orientando e apoiando ao paciente que está sendo acolhido pela casa, ajudando-o promover o conforto físico e emocional.

\section{REFERÊNCIAS}

1 Instituto Nacional de Câncer (INCA). Diretrizes para a detecção precoce do câncer de mama no Brasil [Internet]. Rio de Janeiro; 2015 [acesso em 2018 dez 11]. Disponível em: http: / / www.saude.pr.gov.br/arquivos /File/Deteccao_precoce_CANCER_MA MA_INCA.pdf

2 Instituto Nacional de Câncer (INCA). Estimativa 2018: incidência de câncer no Brasil [Internet]. Rio de Janeiro; 2017 [acesso em 2018 dez 11]. Disponível em: 


\section{JOURNALOF \\ NURSING \\ ANO HEALTH}

http://www1.inca.gov.br/estimativa/ 2018/estimativa-2018.pdf

3. Ministério da Saúde (BR). Controle dos cânceres do colo do útero e da mama [Internet]. $2^{a}$ ed. Brasília; 2013 [acesso em 2018 dez 11]. Disponível em:

http://bvsms. saude.gov.br/bvs/public acoes/controle_canceres_colo_utero_ 2013.pdf

4 Prolla CMD, Silva OS, Oliveira Netto CB, Goldim JR, Prolla PA. Knowledge about breast cancer and hereditary breast cancer among nurses in a public hospital. Rev lat am enfermagem [Internet]. 2015 Feb [cited 2016 Sept 07];23(1):90-7. Available from: http: / / www.scielo.br/scielo.php?scrip $\mathrm{t}=\mathrm{sci}$ _arttext\&pid=S0104-

11692015000100090

5 Instituto Nacional de Câncer (INCA). Câncer de mama: é preciso falar disso [Internet]. $1^{a}$ ed. Rio de Janeiro; 2014 [acesso em $2018 \mathrm{dez}$ 11]. Disponível em:

http: / /bvsms. saude.gov.br/bvs/public acoes/cancer_mama_preciso_falar_dis so.pdf

6 Nascimento FB, Pitta MGR, Rêgo MJBM. Análise dos principais métodos de diagnóstico de câncer de mama como propulsores no processo inovativo. Arq med [Internet]. 2015 dez [acesso em 2018 out 18];29(6):15359. Disponível em: http://www.scielo.mec.pt/scielo.php ?script=sci_arttext\&pid=S0871 $34132015000600003 \&$ lng $=p t$

7 Meira KC, Guimarães RM, Santos J, Cabrelli R. Análise de efeito idadeperíodo-coorte na mortalidade por câncer de mama no Brasil e regiões. Rev panam salud publica [Internet].
ISSN 2236 - 1987

2015 [acesso em 2018 dez
10];37(6):402-8. Disponível em: https://scielosp.org/pdf/rpsp/2015.v 37n6/402-408/pt

8 Ferrari CF, De Abreu EC, Trigueiro TH, Silva MBGM, Kochla KA, Souza SRRK. Orientações de cuidado do enfermeiro para a mulher em tratamento para câncer de mama. Rev enferm ufpe on line [Internet]. 2018 mar [acesso em 2018 jun 02];12(3):676-83. Disponível em: https: / / doi.org/10.5205/1981-8963v12i3a23299p676-683-2018

9 Santos IDL, Alvares RB, Lima NM, Mattias SR, Cestari MEW, Pinto KRTF. Câncer de mama: o apoio recebido no enfrentamento da doença. Rev enferm ufpe on line [Internet]. 2017 ago [acesso em 2018 dez 08];11 Suppl(8):3222-7

10 Bittencourt JFV, Souza IEO. Necessidades de mulheres no enfrentamento do diagnóstico de câncer de mama e do tratamento cirúrgico. Revista de enfermagem da ufjf [Internet]. $2015 \mathrm{dez}$ [acesso em 2018 dez 08];1(2):161-68. Disponível em:

https: / /enfermagem.ufjf.emnuvens.c om.br/enfermagem/article/view/27

11 Bard BA, Cano DS. O papel da rede social de apoio no tratamento de adultos com câncer. Mudanças em psicologia da saúde [Internet]. 2018 [acesso em $2018 \mathrm{dez} 08$ ];26(1):23-33. Disponível em: https://www.metodista.br/revistas/r evistas-

metodista/index.php/MUD/article/vie $\mathrm{w} / 8742 / 6351$

12 Minayo MCS. O desafio do conhecimento: pesquisa qualitativa em 


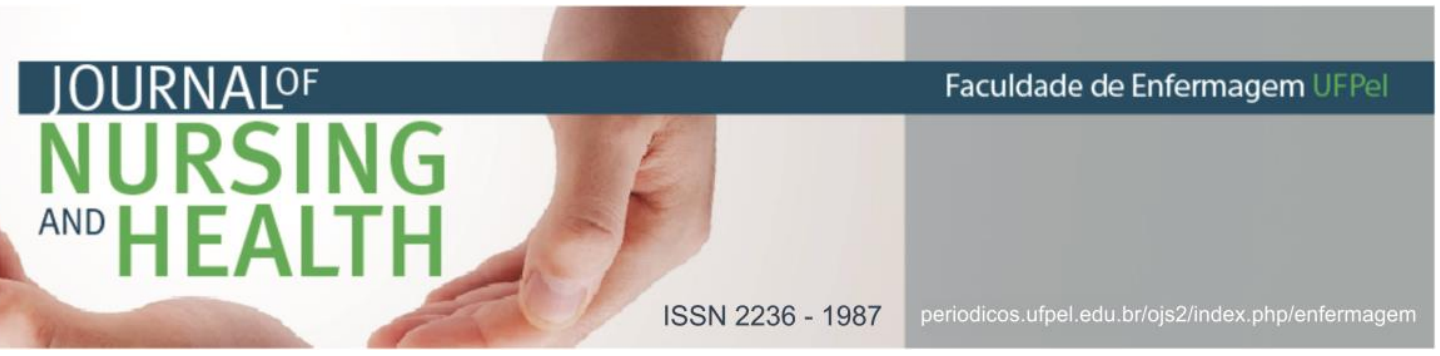

saúde. $14^{\mathrm{a}}$ ed. São Paulo: Hucitec; 2014.

13 Ministério da Saúde (BR). Conselho Nacional de Saúde. Resolução 466, de 12 de dezembro de 2012: diretrizes e normas regulamentadoras de pesquisa envolvendo seres humanos. Brasília; 2012.

14 Ziguer MLPS, De Bortoli CFC, Prates LA. Sentimentos e expectativas de mulheres após o diagnóstico de câncer de mama. Espaç saúde (online) [Internet]. 2016 jul [acesso em 2017 mai 05];17(1):107-12. Disponível em: https://www.researchgate.net/public ation/311635132_Sentimentos_e_expe ctativas_de_mulheres_apos_diagnostic o_de_cancer_de_mama

15 Martins ARB, Ouro TA, Neri $M$. Compartilhando vivências: contribuição de um grupo de apoio para mulheres com câncer de mama. Rev sbph [Internet]. 2015 jun [acesso em 2017 jun 15];18(1):131-51. Disponível em: http://pepsic.bvsalud.org/scielo.php? script $=$ sci_arttext\&pid=S1516$08582015000100007 \& \operatorname{lng}=p t$

16 Ambrosio DCM, Santos MA. Apoio social à mulher mastectomizada: um estudo de revisão. Ciênc saúde colet [Internet]. 2015 mar [acesso em 2017 jun 21];20(3):851-64. Disponível em: http://www.scielo.br/scielo.php?scrip $\mathrm{t}=\mathrm{sci}$ arttext\&pid=S1413-

$81232015000300851 \& \operatorname{lng}=$ en

17 Yoshinari STV, Yoshinari Júnior GH, Masson MV, De Mello LF. Vivência de mulheres frente ao câncer de mama: revisão da literatura brasileira. Rev ciênc saúde [Internet]. 2017 [acesso em 2018 dez 11];7(4):20-5. Disponível http://rcs.fmit.edu.br/index.php/rcsf mit_zero/article/view/707/410

18 Sette CP, Capitão CG. Investigação do suporte social e qualidade de vida em pacientes com câncer. Revista saúde e pesquisa [Internet]. 2018 jan/abr [acesso em 2018 jun 02];11(1):151-62. Disponível em: http://periodicos.unicesumar.edu.br/ index.php/saudpesq/article/view/621 $0 / 3173$

19 de Almeida DR, Gonçalves TR. "Mãos dadas": Experiência da doença em um grupo de apoio ao câncer de mama. Revista prâksis [Internet]. 2015 ago [acesso em 2018 dez 11];2(12):133-45. Disponível em: https: / /www.redalyc.org/pdf/5255/5 25552633013.pdf

20 Martins $M M$, Peres RS. Fatores terapêuticos em grupo de apoio a mulheres com câncer de mama. Psicol saúde doenças [Internet]. 2014 jun [acesso em 2017 Jun 23];15(2):396$408 . \quad$ Disponível em: http://www.scielo.mec.pt/scielo.php ?script=sci_arttext\&pid=S164500862014000200006\&lng=pt

21 Leite LP, Peres RS. Grupos de apoio a mulheres acometidas por câncer de mama: panorama atual. Rev spagesp [Internet]. 2013 [acesso 2018 jun 02];14(1):55-67. Disponível em: http: / / pepsic.bvsalud.org/scielo.php? script $=$ sci_arttext\&pid=S1677$29702013000100007 \& \operatorname{lng}=p t$

22 Canieles IM, Muniz RM, Corrêa ACL, Meincke SMK, Soares LC. Rede de apoio a mulher mastectomizada. Rev enferm ufsm [Internet]. $2014 \mathrm{abr} /$ jun [acesso 2018 jun 02];4(2):450-58. Disponível em: 


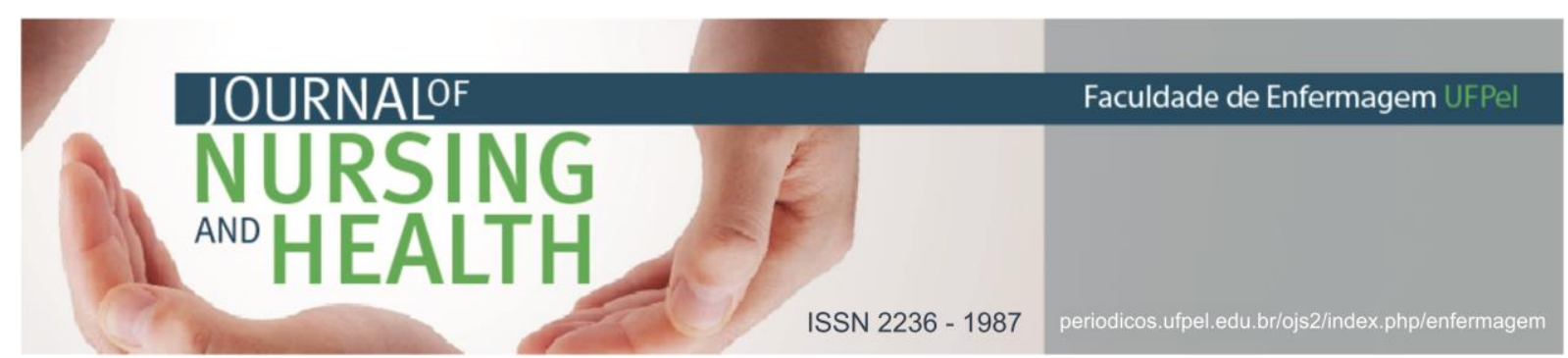

https: / / periodicos.ufsm.br/reufsm/ar ticle/view/10790/pdf

Data de submissão: 18/10/2018

Data de aceite: 11/12/2018

Data de publicação: 14/12/2018 